\title{
ARTICLE
}

Received 24 Oct 2012 | Accepted 12 Apr 2013 | Published 14 May 2013

DOI: $10.1038 /$ ncomms2866

\section{Quantum Faraday and Kerr rotations in graphene}

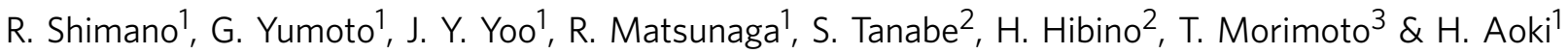

Graphene, a monolayer sheet of carbon atoms, exhibits intriguing electronic properties that arise from its massless Dirac dispersion of electrons. A striking example is the half-integer quantum Hall effect, which endorses the presence of Dirac cones or, equivalently, a non-zero $(\pi)$ Berry's (topological) phase. It is curious how these anomalous features of Dirac electrons would affect optical properties. Here we observe the quantum magneto-optical Faraday and Kerr effects in graphene in the terahertz frequency range. Our results detect the quantum plateaus in the Faraday and Kerr rotations at precisely the quantum Hall steps that hallmark the Dirac electrons, with the rotation angle defined by the fine-structure constant. The robust quantum Hall plateaus in the optical regime, besides being conceptually interesting, may open avenues for new graphene-based optoelectronic applications.

\footnotetext{
${ }^{1}$ Department of Physics, The University of Tokyo, Tokyo 113-0033, Japan. ${ }^{2}$ NTT Basic Research Laboratories, NTT Corporation, Atsugi, Kanagawa 243-0198, Japan. ${ }^{3}$ Condensed Matter Theory Laboratory, RIKEN, Saitama 351-0198, Japan. Correspondence and requests for materials should be addressed to R.S. (email: shimano@phys.s.u-tokyo.ac.jp).
} 
G raphene offers a unique opportunity to study the physics of Dirac fermions in condensed matter ${ }^{1,2}$ that arises from the low-energy band structure with a conical (massless Dirac) dispersion in the effective-mass approximation. The experimental fabrication of monolayer graphene $e^{3}$ has instigated studies of various interesting properties that reflect the nature of massless Dirac fermions, where a notable example is the half-integer quantum Hall effect (QHE) characteristic of Dirac cones $^{4-7}$. Although the quantum phenomena peculiar to graphene have been primarily observed in transport properties, it is gradually beginning to be recognized that the Dirac-fermion nature in graphene has profound effects on dynamical (optical) response, as exemplified by the universal light absorption $\pi \alpha$ $\left(\alpha\right.$, the fine-structure constant) in the near-visible region ${ }^{8}$. The magneto-optical Faraday (Kerr) effect, in which linearly polarized light has its polarization plane rotated when transmitted through (reflected by) a material in the presence of a magnetic field or magnetization, can be thought of as the 'optical-Hall effect' when the phenomenon arises from conducting electrons. Then we can pose a question whether the QHE can be observed in the Faraday and Kerr rotations in graphene; this is a highly nontrivial question, as we do not know whether the degradation of the topological protection of the quantized dc Hall conductivity should entirely wash out the QHE phenomena in the ac regime. Physically, the integer QHE in the dc regime is intimately related to the carrier localization ${ }^{9}$, and how this can be extended to the optical regime is an intriguing question.

Recently, a theoretical investigation has demonstrated the appearance of plateau-like structures in the optical Hall conductivity, which was predicted to be observable in Faraday rotation in the photon energy region as large as the cyclotron energy (that is, inter-Landau-level (LL) transitions) in a conventional nonrelativistic two-dimensional electron gas system ${ }^{10}$. Further, the effect has also been predicted for graphene, where the optical-Hall effect should reflect the peculiar QHE that arises from the massless Dirac dispersion in graphene ${ }^{10}$. The Faraday rotation has also been anticipated for the Dirac fermions in timereversal symmetry broken topological insulators, as a consequence of magnetoelectric coupling, the effect of which can be implemented into the quantized surface Hall conductance ${ }^{11,12}$. Therefore, the Faraday effect in graphene provides an opportunity to test the anticipated optical-Hall effect of Dirac fermions in solids. Although the ac QHE has indeed been observed in a 2DEG in a GaAs heterostructure in the microwave ${ }^{13,14}$ and recently in the quasi-optical (terahertz; THz) frequency ${ }^{15}$ ranges, the Faraday effect in graphene, namely in a Dirac-electron system, in the QHE regime has remained elusive. With recent progress in the fabrication of large-area epitaxial graphene on SiC substrate, the measurement of the Faraday effect in monolayer graphene has been performed in the far-infrared region and been accounted for by the classical Drude model ${ }^{16}$.

Here we observe the $\mathrm{THz}$ Faraday and Kerr rotations in monolayer graphene in the QHE regime. Faraday and Kerr rotations exhibit values defined by the fine-structure constant $\alpha \sim 1 / 137$, as anticipated if one assumes the ac Hall conductivity has an overall value similar to the dc one. ${ }^{17}$ The optical QHE in a one-atom-thick system, while conceptually interesting, is made conspicuous by the present finding that quantum Hall plateaus appearing in the magneto-optical response are anomalously robust in relatively low magnetic fields, which reflects graphene's large LL spacings that characterize the Dirac electron.

\section{Results}

Transport properties characterized by $\mathrm{THz}$ spectroscopy. For the $\mathrm{THz}$ experiments, we have employed a large area $\left(10 \times 10 \mathrm{~mm}^{2}\right)$ monolayer graphene epitaxially grown on the $\mathrm{Si}$ face of $\mathrm{SiC}^{18-20}$.

Figure 1a shows the $532 \mathrm{~nm}$ Raman spectra exhibiting the 2D-band specific to the monolayer graphene ${ }^{21}$. The spatial mapping of this $2 \mathrm{D}$-band in Fig. $1 \mathrm{~b}$ indicates the growth of monolayer graphene with a micrometre scale uniformity (see the Methods section for details). Figure 1c shows the longitudinal optical conductivity spectrum $\sigma_{x x}(\omega)$ of graphene averaged over the spot size of the $\mathrm{THz}$ beam $(1.2 \mathrm{~mm}$ for $1 \mathrm{THz})$ obtained from the transmissiontype $\mathrm{THz}$ time-domain spectroscopy (THz-TDS). All the $\mathrm{THz}$ measurements are performed at $5 \mathrm{~K}$. The spectrum is well fitted by the Drude model, $\sigma_{x x}(\omega)=i D / \pi(\omega+i \Gamma)$, where $\Gamma$ is the damping parameter and $D=\left(v_{\mathrm{F}} e^{2} / \hbar\right) \sqrt{\pi|n|}$ is the Drude weight with $n$ being the carrier density and $v_{\mathrm{F}}=1 \times 10^{6} \mathrm{~m} \mathrm{~s}^{-1}$ the Fermi velocity 22,23 . From the fit, the carrier density is estimated as $n=2.2 \times 10^{11} \mathrm{~cm}^{-2}$ and the corresponding Fermi energy as $\left|E_{\mathrm{F}}\right|=56 \mathrm{meV}$ through the relation $n=E_{\mathrm{F}}^{2} / \pi \hbar^{2} v_{\mathrm{F}}^{2}$. The mobility is estimated as $\mu=v_{\mathrm{F}}^{2} e|| E_{\mathrm{F}} \mid \Gamma=2 \times 10^{4} \mathrm{~cm}^{2} \mathrm{~V}^{-1} \mathrm{~s}^{-1}$ with $\Gamma=$ $7 \mathrm{meV}$. As the coverage of monolayer graphene in the sample is $80 \%$, the Fermi energy estimated here is a value averaged over the spot size of the probe $\mathrm{THz}$ beam.

Magneto-optical Faraday effect. Figure 2a depicts the schematic configuration for the Faraday rotation measurement with THz-TDS (see the Methods section for details). Figure $2 \mathrm{~b}$ defines the sign of the rotation angle with respect to the direction of the magnetic field and the light propagation vector. Figure $2 \mathrm{c}$ shows the Faraday rotation $\theta_{\mathrm{F}}(\omega)$ in a low magnetic field regime below $0.7 \mathrm{~T}$. The magnitude of the Faraday rotation angle is seen to increase with the magnetic field, where the sign of the Faraday rotation in the low frequency limit indicates that the carriers are electrons. In the thinfilm limit, the Faraday rotation angle is expressed as ${ }^{24}$

$$
\theta_{\mathrm{F}}(\omega)=\frac{1}{2} \arg \left[\frac{1+n_{\mathrm{sub}}+Z_{0} \sigma_{+}(\omega)}{1+n_{\mathrm{sub}}+Z_{0} \sigma_{-}(\omega)}\right],
$$

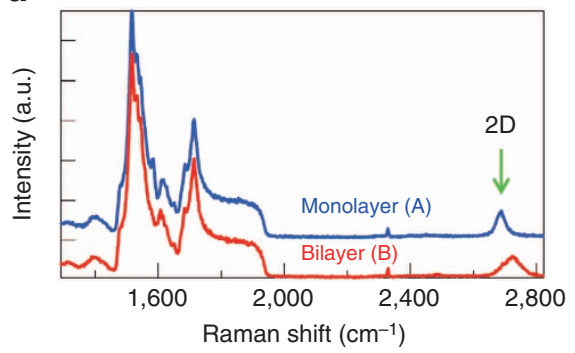

C

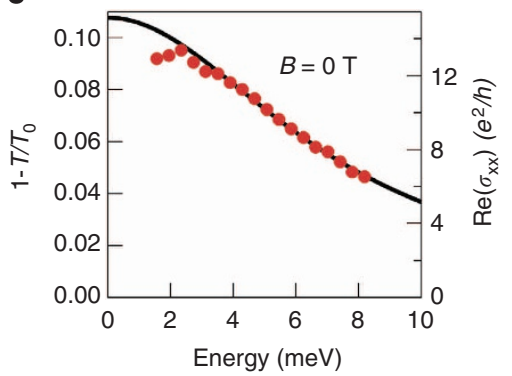

Figure 1 | Raman image of the graphene on SiC and the optical conductivity spectrum. (a) The $532 \mathrm{~nm}$ Raman spectra of the epitaxial graphene on the $\mathrm{SiC}$ substrate at the points $\mathrm{A}$ and $\mathrm{B}$ in the Raman imaging data of (b) taken at the 2D-band of monolayer graphene. The signals below $2,000 \mathrm{~cm}^{-1}$ are mostly attributed to that from SiC substrate. (c) The absorption spectrum (left axis) and the optical conductivity $\sigma_{x x}(\omega)$ spectrum (right axis) of graphene in the $\mathrm{THz}$ range at zero magnetic field. A bare $\mathrm{SiC}$ substrate was used as a reference. The solid curve is the fitted result with the Drude model. 


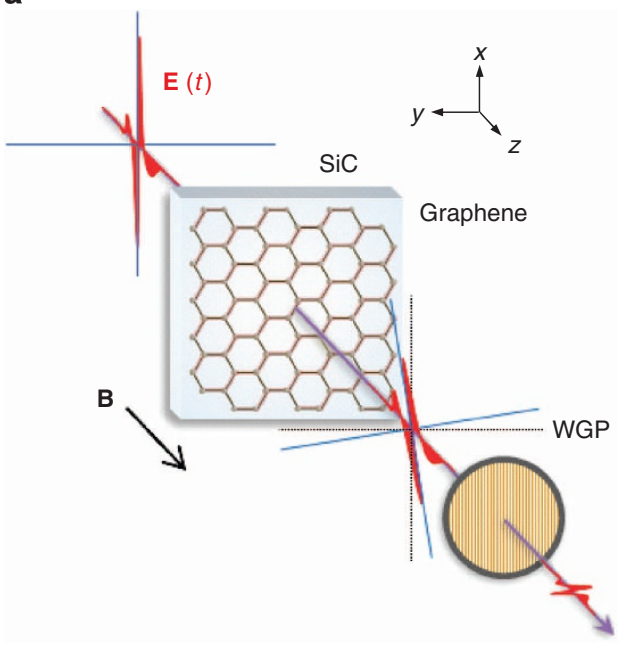

b

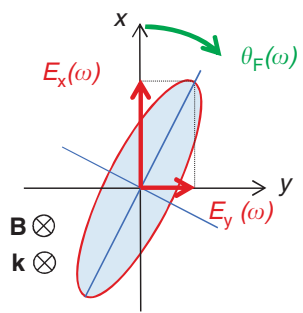

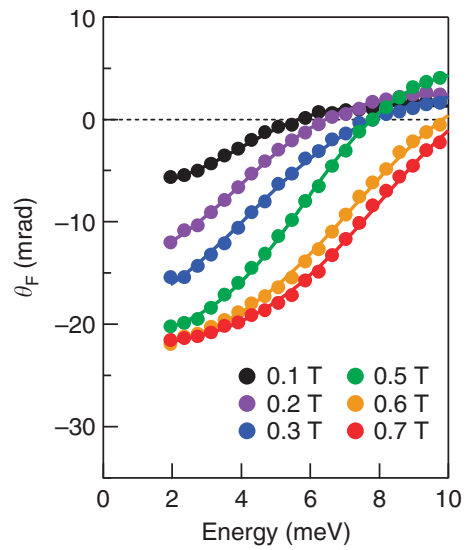

d

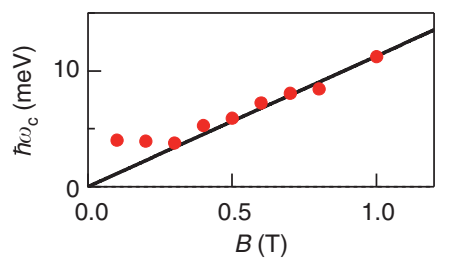

Figure 2 | Faraday rotation in the low magnetic field regime. (a) Schematic of the time-domain $\mathrm{THz}$ polarimetry. The incident THz pulse with the electric field (E-field) linearly polarized along $x$ direction is irradiated onto the sample in a magnetic field $(\boldsymbol{B} / / z)$. The Faraday effect induces the orthogonal ( $y$-) component of the E-field of the transmitted pulse. A wire-grid polarizer (WGP) is placed beyond the sample to measure the parallel and orthogonal components of the transmitted THz E-field, $E_{x}(t)$ and $E_{y}(t)$, respectively. (b) Definition of the Faraday rotation angle $\theta_{\mathrm{F}}(\omega)$. (c) The Faraday rotation spectra (circles) versus photon energy at $5 \mathrm{~K}$ in magnetic fields between 0.1 and $0.7 \mathrm{~T}$. The solid curves are fitted by using the Drude model. (d) The cyclotron frequencies as a function of the magnetic field, obtained from the fitting in (c). The solid line is the calculated one with $E_{\mathrm{F}}=60 \mathrm{meV}$.

where $n_{\text {sub }}(=3.1)$ is the refractive index of the substrate $(\mathrm{SiC})^{25}$, $Z_{0}=377 \Omega$ the vacuum impedance, $\sigma_{ \pm}(\omega)=\sigma_{x x}(\omega) \pm i \sigma_{x y}(\omega)$ the optical conductivity for the right and left circularly polarized light. The Faraday rotation spectra in Fig. $2 \mathrm{c}$ are well fitted by the Drude model (solid curves), according to which the optical Hall conductivity is expressed as $\sigma_{x y}(\omega)=D \omega_{\mathrm{c}} / \pi\left[(\omega+i \Gamma)^{2}-\omega_{c}^{2}\right]$, where $\omega_{\mathrm{c}}=e v_{\mathrm{F}}^{2} B /\left|E_{\mathrm{F}}\right|$ is the cyclotron frequency for graphene ${ }^{22}$. Here, we set $D, \Gamma$ and $\omega_{\mathrm{c}}$ as fitting parameters, where $D$ and $\omega_{\mathrm{c}}$ are not independent. The $\omega_{\mathrm{c}}$ obtained from the fit exhibits a linear dependence on the magnetic field above $0.4 \mathrm{~T}$ as shown in Fig. $2 \mathrm{~d}$, from whose slope the Fermi energy is estimated as $E_{\mathrm{F}}=60 \mathrm{meV}$. This value is consistent with the Drude weight $(D)$ and also with that obtained from the Drude fit to $\sigma_{x x}(\omega)$ at zero field. The linear dependence of the cyclotron frequency indicates that the system is in the classical limit ${ }^{22}$. In low magnetic fields below $0.3 \mathrm{~T}, \omega_{\mathrm{c}}$ obtained from the fit remains nearly constant, whereas the cyclotron resonance should vanish at zero magnetic field. Some excitations not taken into account in the Drude fit, plausibly magnetoplasmons where the cyclotron resonance is coupled with the plasmon localized in the step-and-terrace structure of epitaxial graphene ${ }^{26}$, may be contributing to the optical Hall conductivity spectrum in low magnetic fields.

When we further increase the magnetic field above $1 \mathrm{~T}$, the Faraday rotation turns to decrease as shown in Fig. 3a. Above $3 \mathrm{~T}$ the Faraday rotation becomes frequency-independent, where the cyclotron frequency stays far above the observation frequency. Notably, the rotation angle is pinned to a value of $\theta_{\mathrm{F}}=-5 \mathrm{mrad}$ and hardly changes above $4 \mathrm{~T}$, which is more clearly seen in the magnetic field dependence of the rotation angle at $4 \mathrm{meV}(1 \mathrm{THz})$
(Fig. 3b). The dashed curve represents the classical Drude model as obtained to fit the data in the low magnetic field regime below $0.7 \mathrm{~T}$ with the parameters of $E_{\mathrm{F}}=60 \mathrm{meV}$ and $\Gamma=7 \mathrm{meV}$. Here the calculated curve is multiplied by a factor of 0.7 , which will be discussed later in detail. Although the Drude model well reproduces the data in the low-field regime, a clear deviation is discerned above $3 \mathrm{~T}$ as a shoulder structure. To indicate the magnetic field scale, the inset of Fig. 3b illustrates the LL energies in graphene, $E_{ \pm n}= \pm$ $v_{\mathrm{F}} \sqrt{2 e \hbar B n}(n=0,1,2, \ldots)^{6}$. The first LL exceeds the Fermi energy of $60 \mathrm{meV}$ at $3 \mathrm{~T}$, above which the LL filling of $v=2$ is realized. Therefore, the observed plateau structure in the Faraday rotation can be attributed to the $v=2$ QHE. With the graphene QHE of $\sigma_{x y}=-v e^{2} / h=-2(2 n+1) e^{2} / h$ for $v=2$, the Faraday rotation angle is anticipated to take a value $\theta_{\mathrm{F}}=-4 \alpha /$ $\left(1+n_{\mathrm{sub}}\right)=-7.1 \mathrm{mrad}$, as given by equation (1) neglecting the term $\operatorname{Re}\left(Z_{0} \sigma_{ \pm}\right)\left(\ll 1+n_{\text {sub }}\right)$. This value is close to the observed $-5 \mathrm{mrad}$ in the plateau region.

To confirm the origin of the robust plateau structure in the Faraday rotation, let us compare the result with two kinds of theoretical calculations for the optical Hall conductivity, that is, an exact diagonalization (ED) method ${ }^{10}$ and an equation of motion (EOM) method ${ }^{27}$ (see Method for details). ED, which treats the localization effect, should be accurate in the quantum Hall regime, while it becomes inappropriate, due to the cutoff in the number of included Landau levels, in the classical region for low magnetic fields. EOM method gives a good description for the classical region and a crossover region to the plateau, but gives steeper plateau-toplateau transitions than the ED method. The results with these complementary methods are plotted in Fig. $3 \mathrm{~b}$ as solid (ED) and 
a

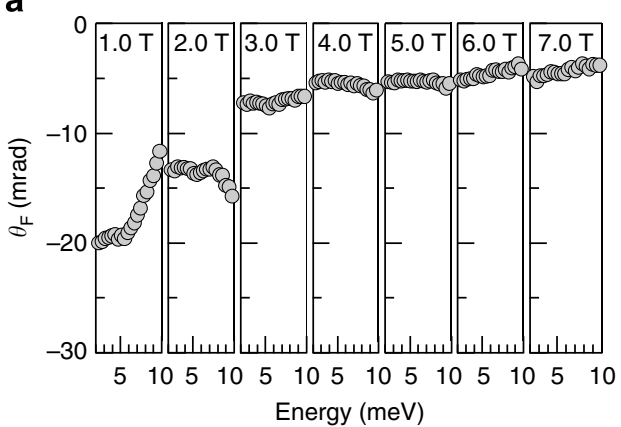

b

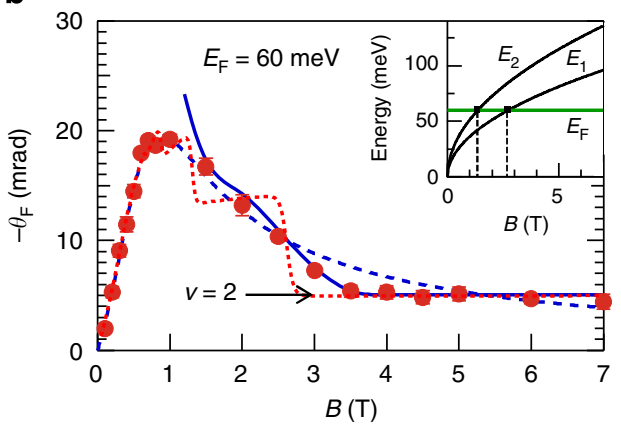

C

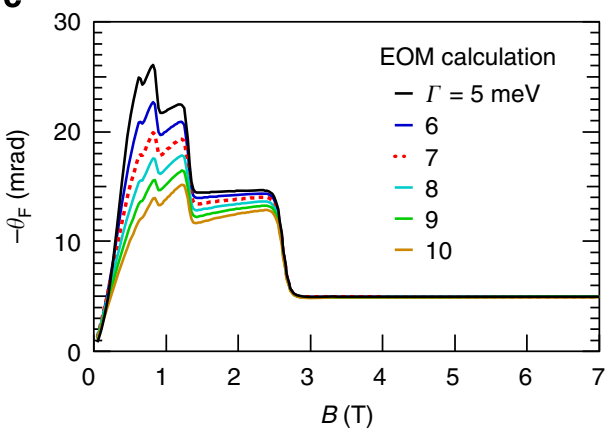

d

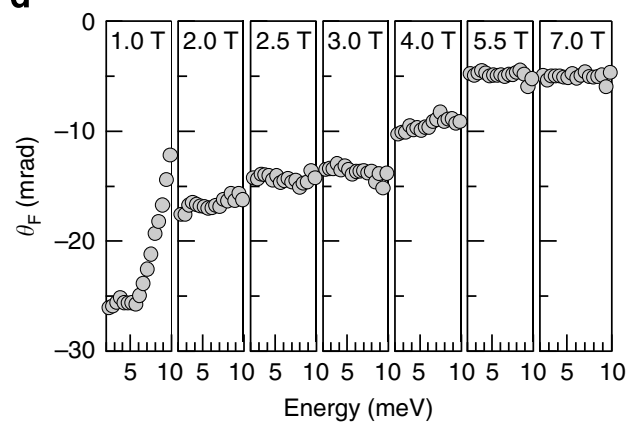

e

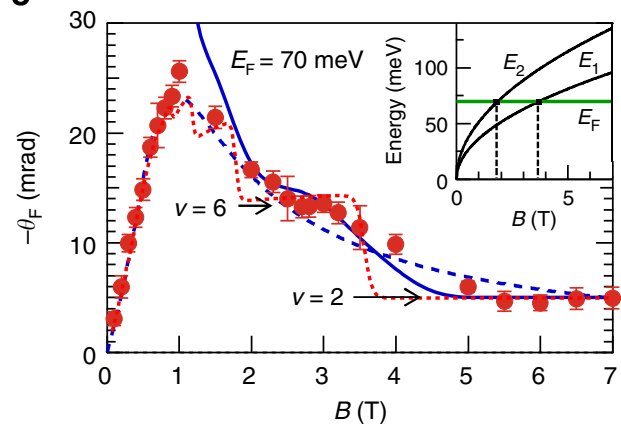

f

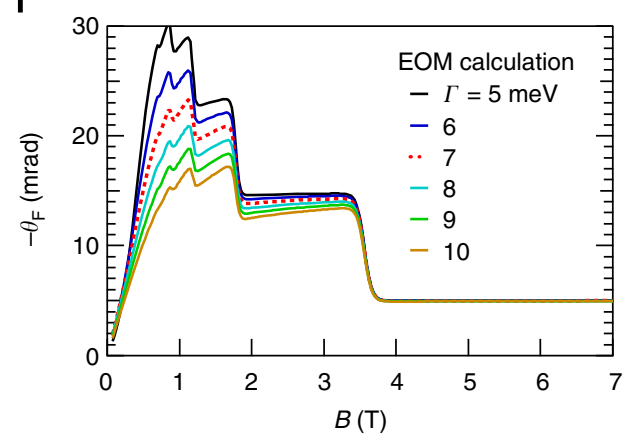

Figure 3 | Faraday rotation in the high magnetic field regime. (a) Faraday rotation spectra in the high magnetic field regime up to $7 \mathrm{~T}$ at $5 \mathrm{~K}$ for the sample with $E_{\mathrm{F}}=60 \mathrm{meV}$. (b) The magnetic field dependence of the Faraday rotation angle at the photon energy of $4 \mathrm{meV}(1 \mathrm{THz})$. Dashed curve is calculated by the Drude model multiplied by a reduction factor of 0.7 (see text for details). Solid curve is the theoretical calculation obtained from the Kubo formula with the ED method, while dotted curve from the EOM method, both for a phenomenological damping parameter $\Gamma=7$ meV. Inset shows the magnetic field dependence of the two LLs in graphene ( $n=1$ and 2) with the green line indicating $E_{\mathrm{F}}=60 \mathrm{meV}$. (c) The EOM calculation for various values of $\Gamma=5-$ $10 \mathrm{meV}$ (see Methods section for details). (d-f) The same plots as a-c, respectively, for the sample with $E_{\mathrm{F}}=70 \mathrm{meV}$.

dotted (EOM) curves with multiplying a factor of 0.7 to adjust the absolute value. Although both results have plateau structures, ED result is seen to be closer to the experimental result.

To further endorse the correspondence, we plot the result for the Faraday rotation in a sample with $E_{\mathrm{F}}=70 \mathrm{meV}$ in Fig. 3d,e. Now, the $v=2$ plateau region shifts to higher magnetic fields, while another plateau appears around $3 \mathrm{~T}$. By comparing the LLs and the Fermi energy (inset of Fig. 3e), the new plateau can be assigned to $v=6$, next in the graphene QHE sequence. Correspondingly, the value of the Faraday rotation on this plateau exhibits about three times as large as that for $v=2$. The result exhibits an excellent agreement with the numerical calculation (solid curve) for both of the plateaus.

Magneto-optical Kerr effect. Having seen the robust quantum Hall plateaus in the Faraday rotation, let us now turn to the magneto-optical Kerr effect in a reflection geometry. As shown in
Fig. 4a,b, the short probe pulse used in the THz-TDS enabled us to detect a delayed second pulse that is multiply reflected inside the substrate after the first main pulse with a separation $\Delta t=2 n_{\text {sub }} L / c$ in time-domain, where $L$ is the thickness of the substrate and $c$ the speed of light. The second pulse experiences the Kerr rotation when it reflects at the interface between the substrate and graphene, while experiences the Faraday effect when it transmits graphene, a geometry which has also been adopted to topological insulators ${ }^{28,29}$. In the small signal limit $\left(\theta_{\mathrm{F}}(\omega), \eta_{\mathrm{F}}(\omega) \ll 1\right)$, the total rotation angle amounts to the sum of the Faraday and Kerr rotations. Thus, we can extract the Kerr rotation from the delayed second pulse by subtracting the Faraday rotation determined from the polarization of the first pulse. The inset of Fig. $4 \mathrm{c}$ shows the Kerr rotation spectra thus obtained for $E_{\mathrm{F}}=70 \mathrm{meV}$. Figure $4 \mathrm{c}$ shows the magnetic field dependence of the Kerr rotation at $1 \mathrm{THz}$, where the Kerr rotation angle exhibits a pinned value of $\theta_{\mathrm{K}}=-15 \mathrm{mrad}$ independent of the magnetic field above $5.5 \mathrm{~T}$. The Kerr rotation caused by the 
a

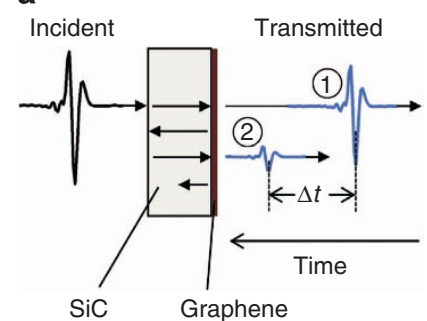

b

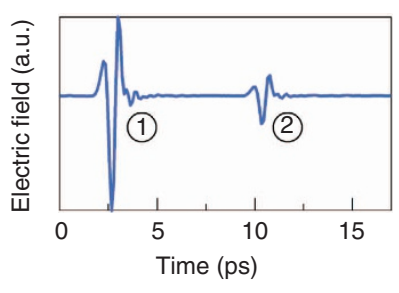

C

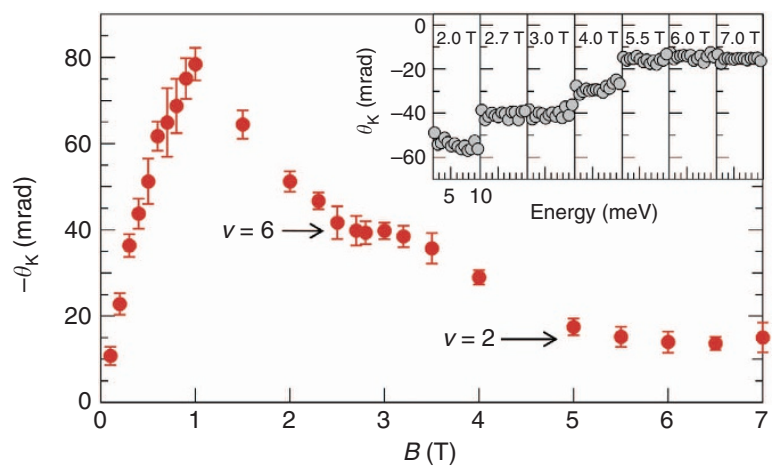

Figure 4 | Magneto-optical Kerr rotation in the monolayer graphene.

(a) Schematic of the transmission of the THz pulse in the sample. (b) The time-domain waveform of the $\mathrm{THz}$ pulse transmitted through the sample. (c) The magnetic field dependence of the Kerr rotation at $1 \mathrm{THz}$ for the sample with $E_{\mathrm{F}}=70 \mathrm{meV}$. The inset shows the Kerr rotation spectrum at the indicated magnetic fields.

reflection at the interface between the substrate and the graphene monolayer is given by

$$
\theta_{\mathrm{K}}(\omega)=-\frac{1}{2} \arg \left[\frac{r_{-}}{r_{+}}\right],
$$

where $r_{ \pm}=\left(n_{\text {sub }}-1-Z_{0} \sigma_{\mp}\right) /\left(n_{\text {sub }}+1+Z_{0} \sigma_{\mp}\right)$ is the complex reflectivity coefficient for right and left circularly polarized light. In the quantum Hall regime $\left(\sigma_{ \pm} \sim \mp i v e^{2} / h\right)$, the Kerr rotation is reduced to $\tan \theta_{\mathrm{K}}(\omega) \sim-4 v \alpha n_{\text {sub }} /\left(n_{\text {sub }}^{2}-1\right)$ and anticipated to exhibit $\theta_{\mathrm{K}}(\omega) \sim-21 \mathrm{mrad}$ for $v=2$. The observed Kerr rotation angle of $\theta_{\mathrm{K}}=-15 \mathrm{mrad}$ matches well with this theoretical value if one assumes the reduction factor of 0.7 for the effective finestructure constant discussed above.

\section{Discussion}

Now let us discuss possible origins of the factor of 0.7 in the observed Faraday rotation. One possibility for the smaller rotation is the existence of electron-hole puddles ${ }^{30,31}$ in the monolayer graphene, which results in the reduction of Faraday rotation. However, the carrier density in the puddles has been estimated to be as low as $10^{9} \mathrm{~cm}^{-2}$ from the previous transport measurements in similar epitaxial graphene samples embedded in an FET structure ${ }^{18}$. Thus, the electron-hole puddles may not be considered as a plausible origin. The second possibility is the adsorption of water molecules on the surface of graphene. The reduction of the 'effective' fine-structure constant has been reported from the transport measurements in graphene covered by a few layers of water molecules ${ }^{32}$. In our experiments, the sample was set in a cryostat filled with $\mathrm{He}$ gas, but not in an ultrahigh vacuum condition, so that adsorption of water molecules in monolayer level was unavoidable. The existence of a buffer layer between the $\mathrm{SiC}$ substrate and graphene (see also Methods section) may also affect the effective dielectric constant in equation $(1)^{33}$. In addition, Raman images (Fig. 1b) indicate that monolayer graphene covers only a part of the total sample area, where the estimated coverage of 0.8 should also reduce the Faraday rotation. Systematic studies, for example, comparing samples with different coverages, will further elucidate the origin of the reduction factor.

To conclude, we have demonstrated the plateau structures and quantum steps in the $\mathrm{THz}$ frequency Faraday and Kerr effects in the monolayer graphene associated with the half-integer QHE. At each plateau region, the rotation angle indeed exhibits a finestructure constant-limited value. In the dc Hall measurement a 'room-temperature QHE' has been observed in graphene ${ }^{34}$, so in this context the quantum magneto-optical effect may possibly appear even at room temperature and suggests the potential functionality of graphene for optical storage devices highly robust against fluctuations in magnetic field and also for an achromatic polarization rotator with very high accuracy and low dissipations in the $\mathrm{THz}$ frequency range. Another interesting extension of the present experiment will be the optical study of the quantum anomalous Hall effect in graphene ${ }^{35,36}$.

\section{Methods}

Sample. Monolayer epitaxial graphene samples were grown on $6 \mathrm{H}-\mathrm{SiC}(0001)$ substrates in an infrared rapid thermal annealing chamber. The $\mathrm{SiC}$ was annealed at $1,500{ }^{\circ} \mathrm{C}$ in $\mathrm{H}_{2}$ gas to clean the surface and then annealed at $1,750{ }^{\circ} \mathrm{C}$ in $\mathrm{Ar}$ gas at a pressure of $\sim 100$ Torr. Although the Raman image in Fig. 1b indicates the micrometre scale uniformity of monolayer graphene, the existence of bilayer graphene is discerned in the residual area (for example, point B in Fig. 1b) as depicted by the Raman spectrum specific to the bilayer graphene (Fig. 1a). Small patches of bilayer graphene and the buffer layer should also exist in the monolyer-graphene dominant area ${ }^{19}$, although they cannot be resolved by the present Raman imaging with a spatial resolution of $1 \mu \mathrm{m}$.

Terahertz time-domain spectroscopy. We adopt the THz-TDS for the polarization spectroscopy in the $\mathrm{THz}$ frequency range. A mode-locked Ti:Sapphire laser with the wavelength of $800 \mathrm{~nm}$, the pulse width of $110 \mathrm{fs}$ and the repetition rate of $76 \mathrm{MHz}$ was used as a light source. THz pulses were emitted from a $p$-type InAs, which is irradiated by the laser pulses. The waveform of the $\mathrm{THz}$ pulse transmitted after the sample was detected by free-space electro-optical sampling with a (110)oriented ZnTe crystal. The sample was set in a split-type superconducting magnet cryostat.

In order to measure the polarization rotation due to the Faraday and Kerr effects, we used free-standing wire-grid polarizers (WGPs). By setting a WGP placed after the sample parallel $(x-)$ or orthogonal $(y-)$ to the incident linear $(x-)$ polarization, we measured the transmitted THz electric fields $E_{x}(t)$ and $E_{y}(t)$, respectively. From the Fourier components of the THz field, $E_{x}(\omega)$ and $E_{y}(\omega)$, the Faraday rotation $\theta_{\mathrm{F}}(\omega)$ and the ellipticity $\eta_{\mathrm{F}}(\omega)$ are obtained through the relation of $E_{y}(\omega) / E_{x}(\omega)=\left(\sin \theta_{\mathrm{F}}+i \eta_{\mathrm{F}} \cos \theta_{\mathrm{F}}\right) /\left(\cos \theta_{\mathrm{F}}-i \eta_{\mathrm{F}} \sin \theta_{\mathrm{F}}\right)$. In the small angle limit, the relation is simplified as $E_{y}(\omega) / E_{x}(\omega) \sim \theta_{\mathrm{F}}(\omega)+i \eta_{\mathrm{F}}(\omega)$. To detect the small Faraday rotation angle, we measured the signals at both the positive and negative magnetic fields as $\theta_{\mathrm{F}}(+B)$ and $\theta_{\mathrm{F}}(-B)$, respectively. The subtraction $\left(\theta_{\mathrm{F}}(+B)-\theta_{\mathrm{F}}(-B)\right) / 2$ eliminates the background signal that arises from the imperfectness of the WGP and enables the detection of small Faraday rotation signal $(\sim \operatorname{mrad})$ from a monolayer graphene. The error bars in Figs $3 b, d, 4 c$ were determined by the s.d. of the measurements repeated ten times. For further details of polarization spectroscopy based on THz-TDS, see Ikebe and Shimano ${ }^{37}$.

Theoretical calculations. We performed two theoretical calculations for the optical Hall conductivity $\sigma_{x y}(\omega)$, ED and EOM methods. ED directly applies the Kubo formula, into which the wavefunctions in disordered systems obtained with diagonalization are plugged (Equation 1 in Morimoto et al. ${ }^{10}$ ), and an ensemble average over impurity configurations is then taken. In the calculation, $E_{\mathrm{F}}$ was fixed (to 60 or $70 \mathrm{meV}$ ) and the disorder potential was introduced by randomly placed long-range scatterers, where we set the potential depth of scatterers so that the width of the cyclotron resonance becomes $7 \mathrm{meV}$ in the plateau region (see Morimoto et al. ${ }^{10}$ for details). ED method should give an accurate description for the plateau region in the high magnetic field regime, as it fully incorporates the Anderson localization effect from which the plateaus originate, but it should become less accurate for low magnetic fields where LLs become dense and the system approaches classical. Namely, a cutoff in the number of included LLs, which we have to introduce in the diagonalization to have finite-dimensional matrices, gives a good approximation for the QHE regime, but is not appropriate for the classical region where LLs become dense. Incidentally, we note that although the calculation is performed for a fixed Fermi energy, a fixed density of electrons gives a similar result for the plateau structure. On the other hand, EOM is developed by Ferreira et al. ${ }^{27}$, where a magneto-optical conductivity is described through EOM 
of the current density and concisely reproduces an expression with Green's function calculations ${ }^{38}$. We have adopted the formula given by equation 55 in Ferreira et al. ${ }^{27}$ with a phenomenological damping parameter $\Gamma=7 \mathrm{meV}$ (Fig. 3b,e), as the EOM method agrees with the Drude model in low magnetic fields. Although EOM gives a good description for the classical region and a crossover region towards the QHE, the plateau-to-plateau transition is steeper than that of ED calculation, as the method does not take account of localization effects. Even if we adopt other values of $\Gamma$ in EOM (Fig. 3c,f), the fits are not improved, and the experimental results in the plateau region are indeed better described by the ED method.

\section{References}

1. Semenoff, G. W. Condensed-matter simulation of a three-dimensional anomaly. Phys. Rev. Lett. 53, 2449-2452 (1984).

2. Haldane, F. D. M. Model for a quantum Hall effect without landau levels: condensed-matter realization of the "Parity Anomaly". Phys. Rev. Lett. 61, 2015-2018 (1988).

3. Geim, A. K. \& Novoselov, K. S. The rise of graphene. Nat. Mat. 6, 183-191 (2007).

4. Novoselov, K. S. et al. Two-dimensional gas of massless Dirac fermions in graphene. Nature 438, 197-200 (2005).

5. Zhang, Y., Tan, Y. -W., Stormer, H. L. \& Kim, P. Experimental observation of the quantum Hall effect and Berry's phase in graphene. Nature 438, 201-204 (2005).

6. Castro Neto, A. H., Guinea, F., Peres, N. M. R., Novoselov, K. S. \& Geim, A. K. The electronic properties of graphene. Rev. Mod. Phys. 81, 109-162 (2009).

7. Goerbig, M. O. Electronic properties of graphene in a strong magnetic field. Rev. Mod. Phys. 83, 1193-1243 (2011).

8. Nair, R. R. et al. Fine structure constant defines visual transparency of graphene. Science 320, 1308 (2008).

9. Aoki, H. \& Ando, T. Effect of localization on the Hall conductivity in the two-dimensional system in strong magnetic fields. Solid State Commun 38, 1079-1082 (1981).

10. Morimoto, T., Hatsugai, Y. \& Aoki, H. Optical Hall conductivity in ordinary and graphene quantum Hall systems. Phys. Rev. Lett. 103, 116803 (2009).

11. Qi, X. -L., Hughes, T. L. \& Zhang, S. -C. Topological field theory of timereversal invariant insulators. Phys. Rev. B 78, 195424 (2008).

12. Tse, W. -K. \& MacDonald, A. H. Magneto-optical Faraday and Kerr effects in topological insulator films and in other layered quantized Hall systems. Phys. Rev. B 84, 205327 (2011).

13. Volkov, V. A. et al. Experimental observation of quantization of the Faraday rotation in a 2D electron system. JETP Lett. 43, 326-329 (1985).

14. Kuchar, F., Meisels, R., Weimann, G. \& Schlapp, W. Microwave Hall conductivity of the two-dimensional electron gas in GaAs- $\mathrm{Al}_{\mathrm{x}} \mathrm{Ga}_{1-\mathrm{x}} \mathrm{As}$. Phys. Rev. B 33, 2965-2967 (1986).

15. Ikebe, Y., Morimoto, T., Masutomi, R., Okamoto, T., Aoki, H. \& Shimano, R. Optical Hall effect in the integer quantum Hall regime. Phys. Rev. Lett. 104, 256802 (2010).

16. Crassee, I. et al. Giant Faraday rotation in single- and multilayer graphene. Nat. Phys. 7, 48-51 (2011).

17. Volkov, V. A. \& Mikhailov, S. A. Quantization of the Faraday effect in systems with a quantum Hall effect. JETP Lett. 41, 476-478 (1985).

18. Tanabe, S., Sekine, Y., Kageshima, H., Nagase, M. \& Hibino, H. Carrier transport mechanism in graphene on $\mathrm{SiC}(0001)$. Phys. Rev. B 84, 115458 (2011).

19. Tanabe, S., Sekine, Y., Kageshima, H., Nagase, M. \& Hibino, H. Half-integer quantum Hall effect in gate-controlled epitaxial graphene devices. Appl. Phys. Exp. 3, 075102 (2010).

20. Hibino, H., Tanabe, S., Mizuno, S. \& Kageshima, H. Growth and electronic transport properties of epitaxial graphene on SiC. J. Phys. D Appl. Phys. 45, 154008 (2012)

21. Ferrari, A. C. et al. Raman spectrum of graphene and graphene layers. Phys. Rev. Lett. 97, 187401 (2006).
22. Ando, T., Zheng, Y. S. \& Suzuura, H. Dynamical conductivity and zero-mode anomaly in honeycomb lattices. J. Phys. Soc. Jpn. 71, 1318-1324 (2002).

23. Orlita, M. et al. Classical to quantum crossover of the cyclotron resonance in graphene: a study of the strength of intraband absorption. N. J. Phys. 14, 095008 (2012).

24. O'Connell, R. F. \& Wallace, G. Ellipticity and Faraday rotation due to a twodimensional electron gas in a metal-oxide-semiconductor system. Phys. Rev. B 26, 2231-2234 (1982).

25. Patrick, L. \& Choyke, W. J. Static dielectric constant of SiC. Phys. Rev. B 2, 2255-2256 (1970).

26. Crassee, I. et al. Intrinsic terahertz plasmons and magnetoplasmons in large scale monolayer graphene. Nano Lett. 12, 2470-2474 (2012).

27. Ferreira, A. et al. Faraday effect in graphene enclosed in an optical cavity and the equation of motion method for the study of magneto-optical transport in solids. Phys. Rev. B 84, 235410 (2011).

28. Hancock, J. N. et al. Surface state charge dynamics of a high-mobility threedimensional topological insulator. Phys. Rev. Lett. 107, 136803 (2011).

29. Valdés Aguilar, R. et al. Terahertz response and colossal Kerr rotation from the surface states of the topological insulator $\mathrm{Bi}_{2} \mathrm{Se}_{3}$. Phys. Rev. Lett. 108, 087403 (2012).

30. Martin, J. et al. Observation of electron-hole puddles in graphene using a scanning single-electron transistor. Nat. Phys. 4, 144 (2008).

31. Zhang, Y., Brar, V. W., Girit, C., Zettl, A. \& Crommie, M. F. Origin of spatial charge inhomogeneity in graphene. Nat. Phys. 5, 722 (2009).

32. Jang, C. et al. Tuning the effective fine structure constant in graphene: opposing effects of dielectric screening on short- and long-range potential scattering. Phys. Rev. Lett. 101, 146805 (2008).

33. Peres, N. M. R. Colloquium: the transport properties of graphene: an introduction. Rev. Mod. Phys. 82, 2673 (2010).

34. Novoselov, K. S. et al. Room-temperature quantum Hall effect in graphene. Science 315, 1379 (2007).

35. Tse, W. -K., Qiao, Z., Yao, Y., MacDonald, A. H. \& Niu, Q. Quantum anomalous Hall effect in single-layer and bilayer graphene. Phys. Rev. B 83, 155447 (2011).

36. Young, A. F. et al. Spin and valley quantum Hall ferromagnetism in graphene. Nat. Phys. 8, 550-556 (2012).

37. Ikebe, Y. \& Shimano, R. Characterization of doped silicon in low carrier density region by terahertz frequency Faraday effect. Appl. Phys. Lett. 92, 012111 (2008).

38. Gusynin, V. P., Sharapov, S. G. \& Carbotte, J. P. Magneto-optical conductivity in graphene. J. Phys. Condens. Matter 19, 026222 (2007).

\section{Acknowledgements}

This research was in part supported by Grant-in-Aid for Scientific Research, No. 23340112 from JSPS.

\section{Author contributions}

G.Y., J.Y., and R.M. performed the THz spectroscopy. S.T. and H.H. fabricated the samples and performed the Raman measurements. G.Y., R.M., J.Y. and R.S. analysed the data. T.M. and H.A. performed theoretical calculations. R.S. and H.A. wrote the paper with substantive feedbacks from all other authors. R.S. conceived the project. All the authors contribute to the interpretation of the results.

\section{Additional information}

Competing financial interests: The authors declare no competing financial interests.

Reprints and permission information is available online at http://npg.nature.com/ reprintsandpermissions/

How to cite this article: Shimano, R. et al. Quantum Faraday and Kerr rotations in graphene. Nat. Commun. 4:1841 doi: 10.1038/ncomms2866 (2013). 